\title{
MODELACIÓN MATEMÁTICA DE TECHOS VERDES EN COLOMBIA: SELECCIÓN DE UN MODELO MEDIANTE LA METODOLOGÍA AHP
}

\section{Selection of a mathematical model through the AHP methodology to model green roofs in Colombia}

\author{
Jeniffer Tabares Catimay ${ }^{1}$, Laura Marely Gallo Martínez², Néstor Alonso Mancipe ${ }^{3}$ \\ ${ }^{1}$ Universidad de La Salle, estudiante del programa de Ingeniería Ambiental y Sanitaria, Colombia. Email: jtabares29@unisalle.edu.co \\ 2 Universidad de La Salle, estudiante del programa de Ingeniería Ambiental y Sanitaria, Colombia. Email: jtabares29@unisalle.edu.co \\ ${ }^{3}$ Universidad Nacional de Colombia. Ingeniero Ambiental y Sanitario. Doctor en Ingeniería Ambiental. Profesor asistente, Colombia. Email: nmanci- \\ pe@unal.edu.co
}

(Recibido21 de Febrero 2019 y aceptado04 de Julio 2019)

\begin{abstract}
Resumen
Los techos verdes (TV) son estructuras que almacenan aguas lluvia, convirtiéndose en una alternativa para el manejo de la escorrentía urbana. Esta investigación se realiza con el fin de seleccionar un modelo matemático para que posteriormente simule la dinámica de TV experimentales construidos en Bogotá, mejorando el proceso de toma de decisiones frente a su implementación en zonas urbanas intertropicales de Colombia al considerar variables meteorológicas, del sustrato y de diseño. Para realizar la selección se empleó la metodología procesos analíticos jerárquicos (AHP por sus siglas en inglés), técnica que emplea operaciones matriciales para tomar decisiones basadas en juicios de valor establecidas por expertos disminuyendo el sesgo generado por la subjetividad de la evaluación. Inicialmente se realiza una consulta bibliográfica determinando modelos matemáticos empleados en la modelación de TV, estos son: SWAM, SWMM 5.1, HYDRUS 1-D, SWAP, SWMS 2-D, MIKE URBAN, WinSLAMM Y SUSTAIN. Posteriormente se califica el desempeño individual de los modelos respecto a tres criterios de selección (datos de entrada, atributos y datos de salida). Esta calificación es evaluada por expertos en hidrología urbana o en Sistemas Urbanos de Drenaje Sostenible (SUDS). Los juicios de valor emitidos por los expertos se utilizan para elaborar matrices pareadas con la metodología AHP, indicando el modelo que mejor cumple los criterios de selección. Se obtuvo que el modelo EPA-SWMM 5.1 cumple satisfactoriamente los requerimientos para modelar techos verdes en Colombia dado que es un software libre, cuenta con un módulo de SUDS, permite modelar eventos de lluvia continuos, entre otras variables. Se desarrolla una lista en orden jerárquico que otros autores podrían usar para seleccionar el modelo que mejor se ajuste a sus necesidades de modelación en TV para condiciones colombianas.
\end{abstract}

Palabras clave: Escorrentía, metodología AHP, modelación matemática, techos verdes.

\begin{abstract}
Green roofs (GR) are engineering structures that stores rainwater as an alternative for urban stormwater management. This research is carried out to select a mathematical model to simulate the experimental TV dynamics from Bogotá, improving the decision-making process regarding GR real implementation in intertropical urban areas of Colombia that considers meteorological, substrate and design variables. A hierarchical analytical method (AHP) is used to select the model for simulating GR. The AHP is a matrix technique that allows a decision-making process based on expert criteria in order to decrease the bias of subjectivity during the assessment. First, a bibliographic review is made to identify GR models to be evaluated: SWAM, SWMM 5.1, HYDRUS 1-D, SWAP, SWMS 2-D, MIKE URBAN, WinSLAMM and SUSTAIN. Then, each model performance is qualified with respect to the selection criteria (Input data, attributes, and output). The value judgments issued by the experts are used to develop paired matrices with the AHP methodology to obtain the model that best meets the selection criteria. The model EPASWMM 5.1 is found to comply with the proposed requirements for modeling GR in Colombia. SWMM is a free software that has a SUDS module and allows continuous events simulation, among other variables. A hierarchical list is developed for other modeling user's decision according to their GR modeling needs.
\end{abstract}

Key words: Runoff, AHP methodology, mathematical modeling, green roofs. 


\section{INTRODUCCIÓN}

Colombia se encuentra ubicada en la zona de confluencia intertropical, produciéndose dos épocas de invierno. En estas épocas las altas precipitaciones incrementan el caudal de escorrentía en especial en zonas urbanas, ya que la impermeabilización de los suelos disminuye los procesos de infiltración, almacenamiento subterráneo y evapotranspiración que ocurren naturalmente en las zonas no urbanizadas [1]. Los picos de escorrentía pueden saturar la red de alcantarillado generando problemas como inundaciones, desbordamientos de ríos y quebradas, pérdidas económicas y humanas, entre otros [2]. Como propuesta para el manejo de la escorrentía urbana y la reducción del riesgo de inundación [3] se han desarrollado sistemas urbanos de drenaje sostenible (SUDS) desde finales de la década de los 70 [4], [5]USA, was estimated from mid-October 2000 through October 2001 based on eddy covariance measurements and statistical modeling from microclimatic data. Measurements were made in $68 \%$ of the hours during the year of study, with $>50 \%$ coverage in all months except December and August. Data were filtered by wind direction and atmospheric turbulence to remove invalid measurements. Analysis of filtered data showed that photosynthetically active radiation (PAR. Entre estos SUDS se encuentran los techos verdes (TV) los cuales cumplen con la función de filtrar, retener y almacenar agua lluvia [6]the hydrologic attenuation of an ecoproductive green roof is assessed using three indicators: lag-time, runoff coefficient and water volume retention. Two types of plants-an herbaceous (Lactuca sativa. Una de las ventajas de los TV, respecto a otros tipos de SUDS es que pueden ubicarse en áreas previamente urbanizadas, como techos y terrazas [3], [7]design, construction and maintenance of Sustainable Drainage Systems (SuDS.

En Colombia la investigación sobre TV se ha centrado en el estudio de montajes experimentales y atenuación de picos de escorrentía. No obstante, el registro continuo de datos en estas investigaciones permite calibrar y validar modelos predictivos que describan el comportamiento hidrológico e hidráulico de los TV en distintos eventos de lluvia. De esta manera, la modelación se convierte en una herramienta de diseño y evaluación para los montajes experimentales.

El objetivo de esta investigación fue seleccionar un modelo matemático que permita realizar un análisis hidro- lógico de un TV en diferentes escenarios dentro de un país intertropical como Colombia. A partir de los resultados de esta investigación se espera realizar la modelación de un módulo de techo verde experimental, cuyos resultados serán abordados en una nueva publicación. La aplicación del proceso analítico jerárquico en la selección del modelo se hizo necesaria para reducir la subjetividad inherente al proceso de selección, esto debido a que existen varias plataformas que permiten modelar el comportamiento hidrológico de distintos tipos de SUDs y cuentan con distintas características que resultan favorables dependiendo del enfoque de la investigación.

En este caso, el modelo debe considerar las variables meteorológicas, variables del sustrato y de diseño, para que los resultados sean útiles en análisis regionales en zonas urbanas de Colombia.

Con base en una revisión bibliográfica se eligieron ocho paquetes de programa (Software) desarrollados por entidades públicas o privadas. La selección se realizó por medio de la metodología procesos analíticos jerárquicos (AHP por sus siglas en inglés) [8]desarrollado por Thomas L. Saaty (The Analytic Hierarchy Process, 1980. Esta metodología está diseñada para cuantificar juicios u opiniones gerenciales sobre la importancia relativa de cada uno de los criterios en conflicto empleados en el proceso de toma de decisión.

La metodología AHP ha sido implementada en otras investigaciones para la toma de decisiones relacionadas con la ubicación de techos verdes. Sangkakool et al. [9] emplearon esta metodología para identificar y cuantificar los factores que influyen en la adopción de techos verdes en áreas urbanas de Tailandia, puesto que los techos son una alternativa para controlar los efectos de isla de calor al mejorar la eficiencia térmica de los edificios. Estos factores fueron evaluados cuantitativamente en un proceso de jerarquía analítica basado en juicios de expertos.

Así mismo Barrero y Ortigoza [10] evaluaron once modelos mediante esta metodología, para encontrar la mejor alternativa y estimar la contaminación por cargas difusas en un área con poca información, esto debido a que en Colombia la información es escasa y de difícil acceso. 


\section{METODOLOGÍA}

Debido a que este trabajo investigativo se basa en el análisis comparativo de distintos modelos matemáticos, la selección se divide en tres fases: consulta bibliográfica de antecedentes, valoración del desempeño de cada modelo para los criterios a evaluar y selección del modelo mediante la aplicación de la metodología AHP.

\subsection{Consulta bibliográfica}

Para la consulta de antecedentes se buscaron artículos científicos sobre modelamiento de techos verdes en bases de datos virtuales como SciELO, Google académico y revistas indexadas. Además, se consultaron bases de datos sobre tesis y trabajos investigativos realizados en la Universidad de Los Andes, La Universidad Nacional y en La Universidad de La Salle. A partir de la información recopilada se seleccionaron algunos modelos que tuvieran características similares y que han sido empleados para la modelación matemática de techos verdes o que por sus características muestran potencial para realizar investigaciones sobre este tema. Posteriormente se realizó una investigación más a fondo sobre las características de estos modelos tales como: parámetros de entrada, tipo de análisis que realizan, escalas de tiempo que manejan, entre otros aspectos relevantes para la evaluación.

\subsection{Valoración del desempeño de cada modelo por parte de expertos}

Para realizar la valoración se acudieron a diferentes expertos investigadores que cuentan con experiencia en techos verdes experimentales y/o en modelación matemática con énfasis en hidrología. Para realizar la valoración se diseñó y envió en formato digital una encuesta que consta de varias preguntas las cuales permiten evaluar el desempeño individual de cada modelo en una escala de valoración. La encuesta se divide en tres secciones: la primera sección corresponde a la información del investigador, en donde debe indicar su nombre, formación profesional y código ORCID; en la segunda sección se le pide al investigador que califique de 1 a 5 la importancia de los criterios de evaluación indicados (sección 2.3.2) siendo 1 "sin importancia" y 5 "importancia máxima"; y en la tercera sección el investigador debe calificar de 1 a 5 el desempeño de cada una de las plataformas para cada subcriterio (sección 2.3.2) de acuerdo con su experiencia y/u otras fuentes de preferencia, siendo $1 \mathrm{el}$ "peor desempeño" y 5 el "desempeño óptimo". Al final, se deja un espacio para que el investigador coloque du- das, sugerencias de otras plataformas y/o comentarios que considere pertinentes.

Adicional a la encuesta se enviaron instrucciones sobre cómo completarla y un archivo que permite contextualizar al investigador sobre las características de cada modelo. El archivo de contextualización incluye una descripción breve de las plataformas a evaluar, las variables que considera, los modelos (ecuaciones) principales que gobiernan la solución numérica de un elemento que pueda representar un techo verde y algunas características relevantes.

\subsection{Metodología AHP}

\subsubsection{Descripción de la metodología AHP}

La metodología AHP (Analytic Hierarchy Process o Proceso Analítico Jerárquico) es una técnica que ayuda a la toma de decisiones multicriterio, para así obtener la mejor alternativa que mejor satisface los criterios que se pretendan evaluar. En esta metodología se realizan comparaciones entre pares de elementos, se cuantifican mediante matrices y se emplean elementos del álgebra matricial para establecer prioridades entre elementos de un nivel con respecto a los de otro [11].

En la figura 1 se puede observar un esquema de esta jerarquización, donde P1, P2 y P3 indican el peso que tiene cada criterio sobre la selección. De modo que es posible que un criterio sea más relevante para la selección o que dos criterios tengan el mismo peso. Las alternativas se evalúan respecto a cada criterio y su desempeño será la suma de los puntajes obtenidos en cada evaluación, la máxima calificación alcanzable para una alternativa sería de 1.0 .

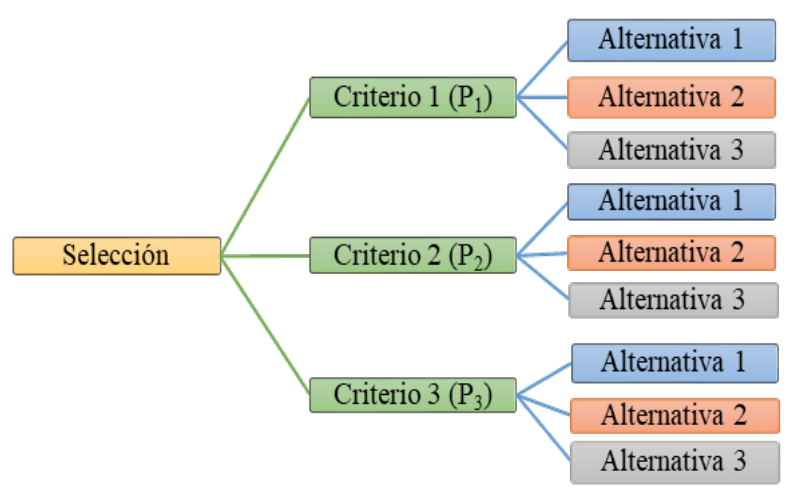

Figura 1. Esquema de jerarquización de la metodología AHP. 
El proceso de jerarquización se inicia construyendo una matriz pareada para los criterios, en esta se analiza la importancia relativa de cada criterio respecto a los demás. Luego se construye una matriz pareada por cada criterio a evaluar en la cual se comparan las alternativas. En estas matrices se cuantifica la comparación entre un criterio o alternativa respecto a otra usando una escala de 1-5 (tabla 1), donde 1 es de igual importancia y 5 importancia extrema de un elemento frente al otro. Los valores pares se utilizan para definir situaciones intermedias [12].

Tabla 1. Escala de preferencias [12]

\begin{tabular}{|c|c|c|}
\hline $\begin{array}{l}\text { Escala } \\
\text { Numérica }\end{array}$ & Escala Verbal & Explicación \\
\hline 1 & Igual importancia & $\begin{array}{l}\text { Los dos elementos contribuyen de igual manera a la propiedad } \\
\text { o criterio }\end{array}$ \\
\hline 2 & Moderadamente más importante & $\begin{array}{c}\text { El juicio y la experiencia favorecen más a un elemento que al } \\
\text { otro }\end{array}$ \\
\hline 3 & $\begin{array}{l}\text { Fuertemente más importante un elemento que } \\
\qquad \text { el otro }\end{array}$ & $\begin{array}{c}\text { El juicio y la experiencia previa favorecen fuertemente a un } \\
\text { elemento frente al otro }\end{array}$ \\
\hline 4 & $\begin{array}{l}\text { Mucho más fuerte la importancia de un } \\
\text { elemento que la del otro }\end{array}$ & $\begin{array}{l}\text { Un elemento domina fuertemente. Su dominación está } \\
\text { probada en práctica }\end{array}$ \\
\hline 5 & $\begin{array}{c}\text { Importancia extrema de un elemento frente al } \\
\text { otro }\end{array}$ & $\begin{array}{l}\text { Un elemento domina al otro con el mayor orden } \\
\text { de magnitud posible }\end{array}$ \\
\hline
\end{tabular}

En las matrices pareadas la calificación se realiza horizontalmente teniendo en cuenta que un criterio o alternativa respecto a si mismo tiene igual importancia $y$, por lo tanto, se le asigna el valor de "1". Para las demás casillas se debe mantener un orden coherente. Por ejemplo, si se tienen dos criterios $O_{i}$ y $P_{j^{\prime}}$, donde el criterio $O_{i}$ es fuertemente más importante que el criterio $P_{j}$ la matriz sería la siguiente:

$$
\left.\begin{array}{ccc} 
& O_{i} & P_{j} \\
O_{i} & 1 & 3 \\
P_{j} & 0.33 & 1
\end{array}\right]
$$

Para cada matriz pareada se debe verificar la consistencia. Se dice que la matriz de comparaciones pareada es consistente cuando los valores dentro de la matriz cumplen que $a_{i j}{ }^{\prime} a_{j k}=a_{i k^{\prime}} \forall \underline{i, j, k}$ siendo $\underline{i, j, k=1,2, \ldots n}$. Esta condición genera una matriz normalizada con columnas idénticas. La evaluación de consistencia se realiza calculando un índice no estadístico denominado razón de consistencia (CR), el cual equivale al cociente entre el índice de consistencia $(\mathrm{Cl})$ y el índice de consistencia aleatorio (RI). La razón de consistencia es aceptable si es menor o igual a 0.1. En caso contrario, quién evalúa debe reconsiderar y modificar sus juicios sobre las comparaciones que realiza ya que esto indica que se favorece en exceso una o varias alternativas. Es decir, hay sesgos en la selección [12]. La forma de calcular estos índices es la siguiente:

$$
\begin{gathered}
C I=\frac{n_{\max }-n}{n-1}(1) \\
R I=\frac{1.98(n-2)}{n}(2) \\
C R=\frac{C I}{R I}(3)
\end{gathered}
$$

Donde, $\mathrm{Cl}$ es el índice de consistencia de la matriz, $\overline{n_{\max }}$ se calcula como la multiplicación de la matriz pareada por los pesos de cada alternativa o criterio de decisión, $n$ es el orden o dimensión de la matriz cuadrada, RI es el índice de consistencia aleatoria y $C R$ es la razón de consistencia de la matriz.

Después de construir las matrices pareadas de criterios y alternativas se procede a normalizarlas. Esta normalización se realiza con el fin de que este proceso genere escalas de razón que capturen la realidad percibida y no sean asignados números arbitrariamente. Para normalizar la matriz se divide cada elemento $\overline{a_{i j}}$ entre la sumatoria de los elementos que componen la

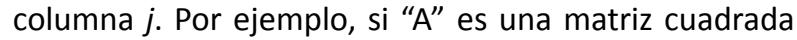
de $n$ filas y columnas, la matriz normalizada " $A$ " " se construiría de la siguiente forma: 


$$
A=\left[\begin{array}{ccc}
a_{11} & \cdots & a_{1 n} \\
\vdots & \ddots & \vdots \\
a_{n 1} & \cdots & a_{n n}
\end{array}\right] \quad A^{\prime}=\left[\begin{array}{ccc}
a_{11}^{\prime} & \cdots & a_{1 n}^{\prime} \\
\vdots & \ddots & \vdots \\
a_{n 1}^{\prime} & \cdots & a_{n n}^{\prime}
\end{array}\right]
$$

Donde,

$$
a_{11}^{\prime}=\frac{a_{11}}{\sum_{i=1}^{n} a_{i 1}}=\frac{a_{11}}{a_{11}+\cdots . .+a_{n 1}}
$$

Posteriormente, de cada matriz se genera un nuevo vector columna denominado "Peso promedio, P", el cual se conforma por los promedios de las filas. Cada elemento del vector columna corresponde al promedio de los elementos de la respectiva fila de la matriz $A^{\prime}$.

$$
P=\left[\begin{array}{l}
P_{11} \\
\cdot \\
\cdot \\
P_{n 1}
\end{array}\right]
$$

En el caso de la matriz de criterios, el vector "Peso promedio" representa el peso de cada criterio en la selección. El criterio con mayor peso será el que defina el resultado. Para las matrices pareadas de alternativas, este vector será la calificación que obtenga cada alternativa para el criterio evaluado. Este vector se calcula de la siguiente forma:

$$
P=\operatorname{Peso} \text { Promedio }\left(A^{\prime}\right)=\left[\begin{array}{ccc}
\operatorname{Prom}\left({a^{\prime \prime}}_{11}\right. & \cdots & \left.a^{\prime}{ }_{1 n}\right) \\
\vdots & \cdot & \vdots \\
\operatorname{Prom}\left(a^{\prime}{ }_{n 1}\right. & \cdots & \left.a^{\prime}{ }_{n n}\right)
\end{array}\right]
$$

Para calcular el puntaje final de cada alternativa se realiza la sumatoria del desempeño ponderado en cada criterio. Es decir, la suma de la multiplicación entre la calificación obtenida y el peso del criterio evaluado.

Para comprendermejor la aplicación del Proceso Analítico Jerárquico en cuanto a la construcción de la matriz pareada, la normalización de las matrices y ponderación de criterios se aconseja revisar el documento realizado por Hurtado [13] y consultar el trabajo realizado por Moreno [12] sobre los fundamentos, la metodología y aplicaciones del Método AHP.

\subsubsection{Selección del modelo}

A partir de la evaluación bibliográfica se eligieron cinco criterios de evaluación, presentados en la Tabla 2. Estos criterios se enfocan en seleccionar un modelo económico de fácil acceso y fácil manejo para el usuario que

\begin{tabular}{|c|c|c|c|}
\hline CRITERIO & SUBCRITERIO & ASPECTO A EVALUAR & OBSERVACIONES/EXPLICACIÓN \\
\hline $\begin{array}{l}\text { Datos de } \\
\text { entrada }\end{array}$ & $\begin{array}{l}\text { Parámetros } \\
\text { considerados }\end{array}$ & $\begin{array}{l}\text { Cantidad e idoneidad de los parámetros } \\
\text { de ingreso considerados }\end{array}$ & $\begin{array}{l}\text { Estos parámetros permitirían ejecutar diferentes eventos } \\
\text { de lluvia. No obstante, se debe considerar la dificultad para } \\
\text { recopilar datos del techo verde antes y durante un evento } \\
\text { de lluvia, Por lo tanto, estas variables serían las mínimas } \\
\text { necesarias para modelar }\end{array}$ \\
\hline \multirow[t]{3}{*}{ Atributos } & $\begin{array}{l}\text { Clasificación } \\
\text { del modelo }\end{array}$ & Distribuido, semi-distribuido, agregado & $\begin{array}{l}\text { En el modelamiento hidrológico de TV se debería } \\
\text { considerar que la estructura se compone de sustrato, } \\
\text { geotextil, capa vegetal y una capa impermeable. Estas } \\
\text { superficies tienen distintas propiedades que afectan el } \\
\text { flujo de la escorrentía. Lo ideal sería que el modelo tenga } \\
\text { en cuenta dichas propiedades en los cálculos. }\end{array}$ \\
\hline & Conexiones & $\begin{array}{l}\text { Posibilidad de conectarse con otros } \\
\text { modelos }\end{array}$ & $\begin{array}{l}\text { Este elemento permitiría importar información desde otras } \\
\text { plataformas o exportar los resultados de la modelación, } \\
\text { ampliando los campos de aplicación de las investigaciones. }\end{array}$ \\
\hline & Costo & $\begin{array}{l}\text { Posibilidad de replicar los resultados } \\
\text { (gratuidad del modelo) }\end{array}$ & $\begin{array}{l}\text { Permitiría que otros investigadores, entidades públicas } \\
\text { o personas naturales, tengan la capacidad de replicar los } \\
\text { resultados de la modelación }\end{array}$ \\
\hline $\begin{array}{l}\text { Datos de } \\
\text { salida }\end{array}$ & $\begin{array}{l}\text { Parámetros } \\
\text { modelados }\end{array}$ & $\begin{array}{l}\text { Cantidad e idoneidad de los parámetros } \\
\text { modelados }\end{array}$ & $\begin{array}{l}\text { Dependiendo del objetivo de la investigación se requieren } \\
\text { ciertos parámetros. Sin embargo, debido a la naturaleza } \\
\text { de los techos verdes se busca modelar parámetros } \\
\text { como escorrentía drenada por el techo, calidad del agua } \\
\text { infiltrada, agua almacenada, humedad del suelo, entre } \\
\text { otros. }\end{array}$ \\
\hline
\end{tabular}
permita determinar el comportamiento hidrológico de un techo verde.

Tabla 2. Criterios de evaluación 
A partir de la información recopilada en la encuesta a expertos investigadores (sección 2.2.) se construyen la matriz de criterios y cinco matrices de alternativas, en las cuales se evalúan los modelos elegidos respecto a los cinco subcrierios presentados en la tabla 2. Para esto se definió una escala de correspondencia (ver Tabla 3) en la cual se relacionan los puntajes asignados por los expertos con la escala de preferencias presentada en la Tabla 1. Para los criterios, la conversión se realiza con base en la diferencia entre los puntajes promedio que obtuvo cada criterio. Por ejemplo, suponiendo que dos criterios obtienen el mismo puntaje promedio, la diferencia sería de 0 y en la matriz pareada se colocaría un valor de 1 en la celda correspondiente dado que ambos criterios tienen igual importancia. Del mismo modo se construyen las matrices pareadas para los modelos evaluados, si dos modelos obtienen el mismo puntaje para un mismo aspecto en la celda respectiva se coloca 1.

Tabla 3. Tabla de conversión para la calificación

\begin{tabular}{|c|c|c|}
\hline Diferencia entre los puntajes & Escala numérica (Tabla 1) & Expresión verbal \\
\hline 0 & 1 & Igual importancia \\
\hline 1 & 2 & Moderadamente más importante \\
\hline 2 & 3 & Fuertemente más importante un elemento que en otro \\
\hline 3 & 4 & Mucho más fuerte la importancia de un elemento que del otro \\
\hline 4 & 5 & Importancia extrema de un elemento frente al otro \\
\hline
\end{tabular}

Cada matriz pareada fue normalizada y se evaluó su consistencia calculando el índice de consistencia $\mathrm{Cl}$, la consistencia aleatoria RI y la razón de consistencia RC como se explicó en la sección 2.3.1. A partir de este proceso se seleccionó el modelo que tiene mejor desempeño en la modelación de techos verdes de acuerdo a los criterios seleccionados.

\section{RESULTADOS}

\subsection{Consulta bibliográfica}

Al realizar la consulta bibliográfica se encontró que en países estacionales se emplean modelos para simular los picos de escorrentía, capacidad de almacenamiento y tiempo de retención de los techos verdes durante eventos de lluvia. La modelación de techos verdes ha sido realizada exitosamente en ciudades como Toronto, Paris, Seattle, Portland, Vancouver, Auckland y New York [14]. Para esto se han empleado modelos de balance hídrico como el Soil Water Apportioning Method (SWAM) [15] y Soil, Water, Atmosphere and Plant model (SWAP)[16], [17]. También se han ejecutado modelos de softwares comerciales como Mike Urban [18], EPA SWMM [19]-[24], HYDRUS 1D [25]-[28] y SWMS 2D [29].
Existen otros modelos que pueden ser implementados para este tipo de análisis, como el software WinSLAMM [30], el cual permite modelar distintos tipos de SUDS y recientemente ha integrado el análisis de techos verdes. El software SUSTAIN ha sido empleado para estudiar otros sistemas de desarrollo de bajo impacto (Low impact development, LID) como depósitos de retención, cunetas verdes y pavimentos permeables [31]. A partir de esta consulta inicial se investigaron las principales características de cada modelo (ver tabla 4) con el fin de ejecutar la comparación pareada mediante la metodología AHP. Cabe resaltar que algunos paquetes comerciales como SewerGEMS, XPSWMM o Infoworks $C$ no se tuvieron en cuenta debido a que no habían sido empleados en investigaciones relacionadas específicamente con modelación de techos verdes [32]. No obstante, se recomienda tenerlos en cuenta en futuras investigaciones que quizás requieran el uso de sistemas de información geográfica o que contemplen el modelado de calidad del agua proveniente de SUDs en sistemas de drenaje urbanos. 
Tabla 4. Resumen de los modelos analizados

\begin{tabular}{|c|c|c|c|c|}
\hline $\begin{array}{c}\text { Nombre del } \\
\text { Modelo }\end{array}$ & Entradas del Modelo & Atributos & Salidas del Modelo & Referencia \\
\hline $\begin{array}{l}\text { SWAM (Soil Water } \\
\text { Apportioning } \\
\text { Method) }\end{array}$ & $\begin{array}{l}\text { Precipitación } \\
\text { Características del suelo } \\
\text { (humedad) } \\
\text { Capacidad de } \\
\text { almacenamiento } \\
\text { Escalas de Tiempo }\end{array}$ & Modelo Agregado & $\begin{array}{l}\text { Diferencia de almacenamientos } \\
\text { Escorrentía } \\
\text { ET }\end{array}$ & [15] \\
\hline $\begin{array}{l}\text { SWMM } \\
\text { (Storm Water } \\
\text { Management } \\
\text { Model) }\end{array}$ & $\begin{array}{l}\text { Precipitación } \\
\text { ET } \\
\text { Temperatura } \\
\text { Variables de Cobertura } \\
\text { Vegetal } \\
\text { Variables del Sustrato. } \\
\text { Variables de geotextil }\end{array}$ & $\begin{array}{l}\text { Modelo semi-distribuido } \\
\text { Conexión con GIS. } \\
\text { Exporta datos a Microsoft } \\
\text { Excel. } \\
\text { Software libre } \\
\text { Desarrollado por USEPA }\end{array}$ & $\begin{array}{l}\text { Escorrentía } \\
\text { Infiltración } \\
\text { Humedades finales del suelo }\end{array}$ & $\begin{array}{l}{[14],[20],} \\
{[21],[23]} \\
{[24],[31]} \\
{[33],[34]} \\
{[35]}\end{array}$ \\
\hline HYDRUS 1-D & $\begin{array}{l}\text { Precipitación } \\
\text { ET } \\
\text { Capacidad de } \\
\text { almacenamiento } \\
\text { Fracción de los tipos de } \\
\text { suelo } \\
\text { Concentraciones de } \\
\text { Soluto }\end{array}$ & $\begin{array}{l}\text { Modelo Distribuido } \\
\text { Exporta datos en formato } \\
\text { texto. } \\
\text { Software libre } \\
\text { desarrollado por USDA ARS }\end{array}$ & $\begin{array}{l}\text { Simulaciones uni y } \\
\text { bidimensionales del agua } \\
\text { Distribución de solutos en medio } \\
\text { saturados }\end{array}$ & {$[25]-[28]$} \\
\hline $\begin{array}{l}\text { SWAP } \\
\text { (Soil, Water, } \\
\text { Atmosphere, } \\
\text { Plant) }\end{array}$ & $\begin{array}{l}\text { Concentraciones de } \\
\text { solutos } \\
\text { Procesos de Distribución } \\
\text { (Dispersión, Adsorción, } \\
\text { convección y } \\
\text { descomposición) }\end{array}$ & $\begin{array}{l}\text { Modelo Agregado. } \\
\text { Software libre } \\
\text { desarrollado por Wageningen }\end{array}$ & $\begin{array}{l}\text { Información para modelos de } \\
\text { transporte (PEARL o ANIMO) } \\
\text { Simula el flujo de calor del suelo }\end{array}$ & {$[16],[17]$} \\
\hline SWMS 2-D & $\begin{array}{l}\text { Concentraciones de } \\
\text { Soluto } \\
\text { Permeabilidad del suelo } \\
\text { Grados de saturación }\end{array}$ & $\begin{array}{l}\text { Modelo Semi-distribuido. } \\
\text { Software libre } \\
\text { desarrollado por USDA ARS }\end{array}$ & $\begin{array}{l}\text { Movimientos de agua y } \\
\text { solutos en medio insaturados, } \\
\text { parcialmente saturados, y } \\
\text { saturados }\end{array}$ & {$[29],[36]$} \\
\hline MIKE URBAN & $\begin{array}{l}\text { Datos meteorológicos } \\
\text { Variables del Suelo }\end{array}$ & $\begin{array}{l}\text { Modelo Semi distribuido } \\
\text { Conexión con plataforma } \\
\text { (SIG) . } \\
\text { Requiere licencia, gratuita } \\
\text { durante } 30 \text { días } \\
\text { desarrollado por DHI }\end{array}$ & $\begin{array}{l}\text { Escorrentía } \\
\text { ET } \\
\text { Infiltración } \\
\text { Flujo superficial } \\
\text { Flujo de desagüe } \\
\text { Balances de masa }\end{array}$ & $\begin{array}{l}{[18],[37],} \\
{[38]}\end{array}$ \\
\hline WinSLAMM & $\begin{array}{l}\text { Precipitación (datos } \\
\text { NOAA) } \\
\text { Dimensiones } \\
\text { Variables del suelo } \\
\text { Vegetación }\end{array}$ & $\begin{array}{l}\text { Modelo Semi distribuido } \\
\text { Conexión con plataformas SIG } \\
\text { (paquete ArcSLAMM) } \\
\text { Costo de la licencia por } \$ 300 \\
\text { dólares } \\
\text { desarrollado por PV } \\
\text { Associates }\end{array}$ & $\begin{array}{l}\text { Escorrentía } \\
\text { Drenado } \\
\text { ET } \\
\text { Análisis del flujo que pasa por } \\
\text { los vertederos }\end{array}$ & {$[30],[39]$} \\
\hline
\end{tabular}

Rev. Ing. Investig. Desarro. Vol.18 (2). pp. 35-46, Julio-Diciembre. 2018, Sogamoso-Boyacá, Colombia. 


\begin{tabular}{|c|c|c|c|c|}
\hline $\begin{array}{l}\text { Nombre del } \\
\text { Modelo }\end{array}$ & Entradas del Modelo & Atributos & Salidas del Modelo & Referencia \\
\hline \multirow{6}{*}{$\begin{array}{l}\text { SUSTAIN } \\
\text { System for } \\
\text { Urban Storm } \\
\text { Water Treatment } \\
\text { and Analysis } \\
\text { Integration }\end{array}$} & Precipitación & \multirow{6}{*}{$\begin{array}{l}\text { Modelo Distribuido o } \\
\text { agregado } \\
\text { Compatible con ArcGIS, } \\
\text { SWMM y HSPF } \\
\text { Software libre. } \\
\text { desarrollado por USEPA }\end{array}$} & Enrutamientos del flujo. & \multirow{6}{*}[31]{,$[40]$} \\
\hline & Variables topográficas. & & Infiltración & \\
\hline & \multirow[t]{4}{*}{ Variables del sustrato } & & Evapotranspiración & \\
\hline & & & $\begin{array}{l}\text { Enrutamiento y eliminación de } \\
\text { contaminantes }\end{array}$ & \\
\hline & & & Sedimentos atrapados & \\
\hline & & & Eliminación de contaminantes & \\
\hline
\end{tabular}

\subsection{Valoración del desempeño de cada modelo por parte de expertos}

Durante la valoración de criterios se obtuvo respuesta de cinco investigadores, que resolvieron la encuesta enviada en formato digital, la universidad a la que están vinculados y el perfil profesional de dichos investigadores se presenta en la tabla 5. Esta información es relevante para el proyecto en la medida en que permite verificar la experiencia que tienen los encuestados en cuanto a investigación sobre SUDS en Colombia.

Tabla 5. Información de los expertos investigados

\begin{tabular}{lll}
\hline \multicolumn{1}{c}{ UNIVERSIDAD } & \multicolumn{1}{c}{ LíNEAS DE INVESTIGACIÓN } \\
\hline $\begin{array}{l}\text { Pontificia Universidad } \\
\text { (Colombia) }\end{array}$ & Javeriana & $\begin{array}{l}\text { Hidrología Urbana, gestión de acueductos y alcantarillado, sistemas sostenibles de drenaje } \\
\text { urbano, aprovechamiento de aguas lluvia. }\end{array}$ \\
& $\begin{array}{l}\text { Hidrología y modelación matemática para la operación y control de sistemas de ingeniería } \\
\text { ambiental conducentes al manejo de los recursos hídricos, gestión de los recursos hídricos, } \\
\text { sistemas Urbanos de Drenaje Sostenible, uso de recursos hidráulicos y teoría de las } \\
\text { singularidades. Encuesta resuelta por dos investigadores. }\end{array}$ \\
$\begin{array}{l}\text { Universidad Nacional de Colombia } \\
\text { (Colombia) }\end{array}$ &
\end{tabular}

Pontifícia Universidad Católica de Hidrología e Hidráulica Urbana, procesos hidrológicos, modelación lluvia-escorrentía chile (Chile)

Hidrología, manejo sostenible de sistemas de abastecimiento y aguas residuales y pluviales, caracterización, modelación, análisis y sostenibilidad de hidro sistemas y ecosistemas

Universidad de los Andes (Colombia)

\subsection{Selección del modelo}

De acuerdo a lo explicado en la metodología, se construyó la matriz de criterios (Tabla 6) y se calculó su matriz normalizada (Tabla 7).

Tabla 6. Matriz de Criterios de evaluación

\begin{tabular}{cccc}
\hline $\begin{array}{c}\text { Criterios de } \\
\text { Evaluación }\end{array}$ & $\begin{array}{c}\text { Datos de } \\
\text { Entrada }\end{array}$ & Atributos & $\begin{array}{c}\text { Datos de } \\
\text { Salida }\end{array}$ \\
\hline Datos de Salida & 0.33 & 0.5 & 1 \\
Atributos & 0.5 & 1 & 2 \\
Datos de Entrada & 1 & 2 & 3 \\
\hline
\end{tabular}

Tabla 7. Matriz de Criterios de evaluación normalizada

\begin{tabular}{ccccc}
\hline $\begin{array}{c}\text { Criterios de } \\
\text { Evaluación }\end{array}$ & $\begin{array}{c}\text { Datos } \\
\text { de } \\
\text { Entrada }\end{array}$ & Atributos & $\begin{array}{c}\text { Datos } \\
\text { de } \\
\text { Salida }\end{array}$ & $\begin{array}{c}\text { Peso } \\
\text { promedio }\end{array}$ \\
\hline $\begin{array}{c}\text { Datos de } \\
\text { Salida }\end{array}$ & 0.182 & 0.14 & 0.17 & 0.164 \\
\hline Atributos & 0.273 & 0.29 & 0.33 & 0.297 \\
\hline $\begin{array}{c}\text { Datos de } \\
\text { Entrada }\end{array}$ & 0.545 & 0.57 & 0.50 & 0.539 \\
\hline & & & $\Sigma$ & 1.000 \\
\hline
\end{tabular}

En la asignación de valores los expertos investigadores les dieron prioridad a los datos de entrada respecto a los atributos y a los datos de salida debido a que el 
objetivo es modelar el comportamiento hidrológico de los techos verdes a partir de la información disponible. Lo anterior puede verse reflejado en el valor del peso promedio resaltado en color azul, el cual corresponde a 0.539 para los datos de entrada, interpretándose como el $53.9 \%$ de influencia.

El siguiente criterio de mayor importancia fueron los atributos del modelo, debido a que la gratuidad del modelo y la posibilidad de integrar el modelo con otro software que incluya el uso de sistemas de información geográfica o poder exportar los datos, resultaría muy útil para ampliar el campo de aplicación en futuras investigaciones.

Posteriormente, para la construcción de las matrices se tomaron en cuenta los subcriterios mencionados en la Tabla 2 de criterios de evaluación. Para cada elemento se evaluó que el modelo cumpliera o no con la preferencia especificada en dicha tabla de acuerdo a los evaluado por los expertos.

De forma ilustrativa a continuación se presenta la matriz pareada del subcriterio "Parámetros considerados" que corresponden a los datos de entrada (Tabla 8), su matriz normalizada (Tabla 9) y los parámetros de consistencia calculados para esta matriz (Tabla 10).
Tabla 8. Matriz pareada para el elemento de Parámetros considerados"

\begin{tabular}{llllllllll}
\hline \multicolumn{10}{c}{ Parámetros Considerados (A) } \\
\hline SWAM & 1.0 & 0.5 & 0.5 & 1.0 & 1.0 & 1.0 & 1.0 & 1.0 & 0.83 \\
SWMM & 2.0 & 1.0 & 1.0 & 2.0 & 2.0 & 1.0 & 2.0 & 1.0 & 1.38 \\
HYDRUS 1-D & 2.0 & 1.0 & 1.0 & 2.0 & 2.0 & 1.0 & 2.0 & 1.0 & 1.38 \\
SWAP & 1.0 & 0.5 & 0.5 & 1.0 & 1.0 & 1.0 & 1.0 & 1.0 & 0.83 \\
SWMS 2-D & 1.0 & 0.5 & 0.5 & 1.0 & 1.0 & 0.5 & 1.0 & 0.5 & 0.69 \\
MIKE URBAN & 1.0 & 1.0 & 1.0 & 1.0 & 2.0 & 1.0 & 2.0 & 1.0 & 1.17 \\
WINSLAMM & 1.0 & 0.5 & 0.5 & 1.0 & 1.0 & 0.5 & 1.0 & 0.5 & 0.69 \\
SUSTAIN & 1.0 & 1.0 & 1.0 & 1.0 & 1.0 & 1.0 & 2.0 & 1.0 & 1.09 \\
Sumatoria & 10 & 6.0 & 6.0 & 10 & 11 & 7.0 & 12 & 7.0 & 8.1 \\
\hline
\end{tabular}

Las matrices pareadas para cada elemento considerado pueden solicitarse al correo del autor para correspondencia.

El resultado final de este proceso de jerarquización se presenta en la tabla 10, las columnas corresponden al vector "Peso promedio" de las matrices de alternativas generadas. Se debe tener en cuenta que la gratuidad del software, relación con otros modelos y la clasificación del modelo son subcriterios que pertenecen al criterio de "Atributos". Por lo cual fueron promediados para obtener el puntaje correspondiente a dicho criterio. Para el cálculo del puntaje final se multiplica el valor de la columna por el peso de cada criterio y se suman, por lo tanto, es un puntaje ponderado.

Tabla 9. Matriz de "Parámetros considerados" normalizada.

\begin{tabular}{|c|c|c|c|c|c|c|c|c|c|}
\hline \multicolumn{10}{|c|}{ Parámetros Considerados - Normalizada } \\
\hline 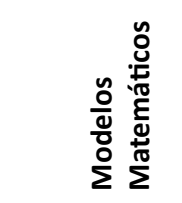 & $\sum_{n}$ & $\sum_{n}$ & 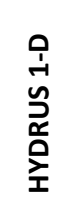 & $\sum_{n}^{a}$ & 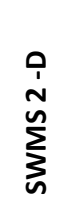 & 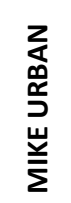 & 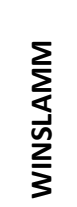 & 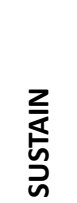 & 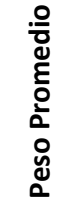 \\
\hline SWAM & 0.10 & 0.08 & 0.08 & 0.10 & 0.09 & 0.14 & 0.08 & 0.14 & 0.10 \\
\hline SWMM & 0.20 & 0.17 & 0.17 & 0.20 & 0.18 & 0.14 & 0.17 & 0.14 & 0.17 \\
\hline HYDRUS 1-D & 0.20 & 0.17 & 0.17 & 0.20 & 0.18 & 0.14 & 0.17 & 0.14 & 0.17 \\
\hline SWAP & 0.10 & 0.08 & 0.08 & 0.10 & 0.09 & 0.14 & 0.08 & 0.14 & 0.10 \\
\hline SWMS 2-D & 0.10 & 0.08 & 0.08 & 0.10 & 0.09 & 0.07 & 0.08 & 0.07 & 0.09 \\
\hline MIKE URBAN & 0.10 & 0.17 & 0.17 & 0.10 & 0.18 & 0.14 & 0.17 & 0.14 & 0.15 \\
\hline WINSLAMM & 0.10 & 0.08 & 0.08 & 0.10 & 0.09 & 0.07 & 0.08 & 0.07 & 0.09 \\
\hline SUSTAIN & 0.10 & 0.17 & 0.17 & 0.10 & 0.09 & 0.14 & 0.17 & 0.14 & 0.13 \\
\hline
\end{tabular}

Rev. Ing. Investig. Desarro. Vol.18 (2). pp. 35-46, Julio-Diciembre. 2018, Sogamoso-Boyacá, Colombia. 
Tabla 10. Indicadores de consistencia para la matriz de "Parámetros considerados"

\begin{tabular}{cc}
\hline $\mathbf{n}_{\text {Max }}$ & $\mathbf{8 . 0 4 7}$ \\
\hline $\mathbf{n}$ (dimensión de la matriz) & 8 \\
Índice de consistencia (CI) & 0.007 \\
Consistencia Aleatoria (RI) & 1.485 \\
Razón de consistencia (RC) & 0.005 \\
$\quad$ Conclusión & \\
Inconsistencia aceptable & \\
(La matriz no es sesgada) & \\
\hline
\end{tabular}

Como parte de las observaciones realizadas por los expertos se encuentra que la idoneidad del modelo depende en gran medida del objetivo de la modelación, puesto que algunos modelos hidrológicos tienen gran desempeño calculando la cantidad de agua, pero pueden tener menor calificación al considerar el flujo de contaminantes a través del techo o la calidad de la escorrentía que se genera. De la misma forma, algunos comentaron que se debería tener en cuenta la facilidad para manejar la plataforma, modificarla de acuerdo a las necesidades de la investigación y para recopilar los datos de entrada, dado que esto influye en gran medida al momento de seleccionar un modelo para realizar una investigación.

En la Tabla 11 se presentan los puntajes obtenidos por cada modelo. El mejor desempeño lo tuvo el modelo desarrollado por la USEPA SWMM, seguido de HYDRUS 1D y SUSTAIN. Estos modelos tienen en común que son gratuitos y permitirían modelar la retención de agua lluvia en distintas escalas de tiempo. SWMM y HYDRUS, al haber sido diseñados por la USEPA, son gratuitos y permiten exportar los datos a otros formatos, esto representa una ventaja para el criterio de "atributos".

Tabla 11. Puntaje de los modelos evaluados

\begin{tabular}{|c|c|c|c|c|}
\hline \multirow{3}{*}{$\begin{array}{c}\text { Modelo } \\
\text { Matemático }\end{array}$} & \multicolumn{3}{|c|}{ Criterio } & \multirow{3}{*}{$\begin{array}{l}\text { Puntaj } \\
\text { Total }\end{array}$} \\
\hline & $\begin{array}{l}\text { Datos de } \\
\text { Entrada }\end{array}$ & Atributos & $\begin{array}{l}\text { Datos de } \\
\text { Salida }\end{array}$ & \\
\hline & \multicolumn{3}{|c|}{$(0.297)$} & \\
\hline SWMM & 0.171 & 0.177 & 0.153 & 0.170 \\
\hline HYDRUS 1-D & 0.171 & 0.183 & 0.092 & 0.162 \\
\hline SUSTAIN & 0.135 & 0.188 & 0.166 & 0.155 \\
\hline MIKE URBAN & 0.146 & 0.100 & 0.166 & 0.135 \\
\hline WINSLAMM & 0.085 & 0.097 & 0.166 & 0.102 \\
\hline SWAP & 0.103 & 0.099 & 0.071 & 0.097 \\
\hline SWAM & 0.103 & 0.060 & 0.111 & 0.092 \\
\hline SWMS 2-D & 0.085 & 0.097 & 0.077 & 0.087 \\
\hline & & & & 1.000 \\
\hline
\end{tabular}

\section{DISCUSIÓN}

Para la matriz de parámetros considerados (Datos de entrada) los modelos que tuvieron el mayor puntaje fueron SWMM Y HYDRUS 1D, con 0.17 y 0.162 respectivamente. La principal ventaja de estos modelos es que para realizar la modelación sólo requieren parámetros climatológicos, dimensiones del techo verde y características del sustrato. Algunos expertos encuestados argumentaron que a menor cantidad de parámetros de ingreso es más factible poder ejecutar el modelo utilizando valores reales medidos en campo. Los modelos que tuvieron menor desempeño en este parámetro fueron SWMS 2D y WinSLAMM. El primero no tiene en cuenta la composición del sustrato ni la vegetación presente en el techo verde, mientras que WinSLAMM obtiene los datos climatológicos principalmente del NOAA (National Oceanic and Atmospheric Administration) y se centra en el transporte de contaminantes en redes de drenaje urbano lo cual no se podría considerar como una prioridad en la modelación del comportamiento hidrológico del techo verde.

Para la matriz de clasificación del modelo la mayoría tuvieron el mismo puntaje excepto SWAM, el cual obtuvo el puntaje más bajo (0.057) ya que es un modelo agregado y no considera la variación entre las propiedades del suelo. Por otro lado, HYDRUS y SUSTAIN tuvieron una mejor calificación al ser modelos distribuidos. Respecto a la posibilidad de conectarse con otros modelos o software, los modelos que no presentan esta posibilidad son SWAM, SWAP y SWMS 2D. En cuanto a la gratuidad del modelo, Mike Urban y WinSLAMM requieren del pago de una licencia para su uso, mientras que SWAM es un modelo que requiere ser solicitado a los autores, por lo que se consideraría que no es de uso libre. En la matriz de parámetros modelados, HYDRUS, SWAP y SWMS 2D tuvieron una baja calificación relativa, ya que los demás modelos permiten determinar el agua almacenada dentro del techo verde y modelar la escorrentía en función del tiempo.

La razón de consistencia de las matrices permite asegurar que hay coherencia entre los valores asignados para cada modelo respecto a los demás. Si un modelo tiene una calificación de 1 respecto a otro, ambos modelos deben tener la misma calificación respecto a los demás modelos. La inconsistencia puede deberse a 
que fue necesario redondear los números decimales al calcular la diferencia entre los puntajes de los modelos. Al haber empelado encuestas con escalas numéricas fue fácil construir las matrices pareadas y en todos los casos se obtuvieron valores muy bajos de $\mathrm{RC}(<0.1)$. Esto indica que no hay sesgo en la calificación. Por otro lado, la matriz que tuvo el valor más alto de RC fue la de "Costo" con un valor de 0.015. La principal dificultad para calificar los modelos fue tener en cuenta varias preferencias en un mismo subcriterio sin ser subjetivos. Sin embargo, en todos los casos la razón de consistencia tuvo un valor aceptable.

\section{CONCLUSIONES}

En conclusión, el modelo que tuvo el mejor puntaje respecto a los criterios evaluados fue SWMM 5.1, ya que su módulo de LID-Control permite modelar los techos verdes considerando variables de vegetación, variables del sustrato, varios eventos de lluvia continuos además de ser un software libre. Sin embargo, hubo otros modelos, como HYRDUS 1-D, SUSTAIN Y MIKE URBAN, que tuvieron gran desempeño en esta evaluación y que por sus características permitirían realizar investigaciones amplias e integrales sobre este tipo de SUDS en zonas intertropicales.

\section{AGRADECIMIENTOS}

Manifestamos nuestros sinceros agradecimientos a los investigadores por su dedicación y tiempo empleado para desarrollar la encuesta suministrada. A la Universidad de La Salle por su apoyo y su compromiso con la formación académica y personal de los profesionales.

\section{REFERENCIAS}

[1] M. Molina, L. Gutiérrez, and J. Salazar, "Sistema Urbanos de Drenaje Sostenible," Ambient. Secr. Dist. Medio, 2011.

[2] A. Torres, S. Méndez-Fajardo, Á. P. Gutiérrez Torres, and S. Sandoval, "Quality of Rainwater Runoff on Roofs and Its Relation to Uses and Rain Characteristics in the Villa Alexandra and Acacias Neighborhoods of Kennedy, Bogota, Colombia," J. Environ. Eng., 2013.

[3] B. Woods-Ballard, R. Kellagher, P. Martin, C. J. R. Bray, and P. Shaffer, The SUDS manual - UK. 2007.

[4] D. Butler and J. Davies, Urban Drainage. 2011.
[5] J. Niemczynowicz, "Urban hydrology and water management - present and future challenges," Urban Water, 1999.

[6] N. Oviedo and A. Torres, "Hydric Attenuation and Hydrological Benefits for Implementing Productive Green Roof in Soacha, Colombia," Ing. y Univ., 2014.

[7] H. F. Castletona, V. Stovinb, S. B. M. Beckc, and J.B. Davisonb, "Green roofs; building energy savings and the potential for retrofit," Energy Build., 2010.

[8] T. L. Saaty and J. Wiley, "Proceso De Análisis Jerárquico (Ahp)," Rev. Digit. UMMSM, 2009.

[9] T. Sangkakool, K. Techato, R. Zaman, and T. Brudermann, "Prospects of green roofs in urban Thailand - A multi-criteria decision analysis," J. Clean. Prod., 2018.

[10] L. Katherine, O. Rojas, L. Fernanda, and B. Chimbi, "ANÁLISIS EXPLORATORIO DE MODELOS PARA LA ESTIMACIÓN DE LA CONTAMINACIÓN POR CARGAS DIFUSAS EN UN ÁREA DE ESTUDIO CON INFORMACIÓN ESCASA."

[11] Juan C. Osorio y Juan P. O, "El Proceso De Análisis Jerárquico (Ahp) Y La Toma De Decisiones Multicriterio. Ejemplo De Aplicación.," Sci. Tech., 2008.

[12] J. Moreno, "El Proceso Analítico Jerárquico ( AHP). Fundamentos, metodología y aplicaciones," Univ. Zaragoza, 2002.

[13] G. B. Toskano Hurtado, “EL PROCESO DE ANÁLISIS JERARQUICO (AHP) COMO HERRAMIENTA PARA LA TOMA DECISIONES EN LA SELECCIÓN DE PROVEEDORES," 2005.

[14] S. S. Cipolla, M. Maglionico, and I. Stojkov, "A longterm hydrological modelling of an extensive green roof by means of SWMM," Ecol. Eng., 2016.

[15] R. Hakimdavar, P. J. Culligan, A. Guido, and W. R. McGillis, "The Soil Water Apportioning Method (SWAM): An approach for long-term, low-cost monitoring of green roof hydrologic performance," Ecol. Eng., vol. 93, pp. 207-220, Aug. 2016.

[16] J. G. Kroes, J. G. Wesseling, and J. C. Van Dam, “Integrated modelling of the soil-water-atmosphere-plant system using the model SWAP 2.0 an overview of theory and an application," in Hydrological Processes, 2000.

[17] J. Wesseling, J. G. Kroes, and K. Metselaar, "Global sensitivity analysis of the Soil-Water-Atmosphere-Plant (Swap) model," Wageningen, SC-DLO, 1998. Rep. 160, 70 pp, 1998.

[18] L. Locatelli, O. Mark, P. S. Mikkelsen, K. Arnbjerg-Nielsen, M. Bergen Jensen, and P. J. Binning, 
"Modelling of green roof hydrological performance for urban drainage applications," J. Hydrol., 2014.

[19] K. Alfredo, F. Montalto, and A. Goldstein, "Observed and Modeled Performances of Prototype Green Roof Test Plots Subjected to Simulated Lowand High-Intensity Precipitations in a Laboratory Experiment," J. Hydrol. Eng., 2010.

[20] E. Burszta-Adamiak and M. Mrowiec, "Modelling of Green roofs' hydrologic performance using EPA's SWMM," Water Sci. Technol., 2013.

[21] A. Palla and I. Gnecco, "Hydrologic modeling of Low Impact Development systems at the urban catchment scale," J. Hydrol., 2015.

[22] A. Bonoli, A. Conte, M. Maglionico, and I. Stojkov, "Green roofs for sustainable water management in urban areas," Environ. Eng. Manag. J., 2013.

[23] G. Krebs, T. Kokkonen, M. Valtanen, H. Setälä, and H. Koivusalo, "Spatial resolution considerations for urban hydrological modelling," J. Hydrol., 2014.

[24] P. A. Versini, D. Ramier, E. Berthier, and B. de Gouvello, "Assessment of the hydrological impacts of green roof: From building scale to basin scale," J. Hydrol., 2015.

[25] R. Hakimdavar, P. J. Culligan, M. Finazzi, S. Barontini, and R. Ranzi, "Scale dynamics of extensive green roofs: Quantifying the effect of drainage area and rainfall characteristics on observed and modeled green roof hydrologic performance," Ecol. Eng., 2014.

[26] R. N. Hilten and T. M. Lawrence, "Using green roofs and other BMPs to reduce the need for stormwater retention capacity requirements," in Low Impact Development: New and Continuing Applications - Proceedings of the 2nd National Low Impact Development Conference 2007, 2008.

[27] A. Palla, I. Gnecco, and L. G. Lanza, "Compared performance of a conceptual and a mechanistic hydrologic models of a green roof," Hydrol. Process., 2012.

[28] H. peng Qin, Y. nuan Peng, Q. ling Tang, and S. L. Yu, "A HYDRUS model for irrigation management of green roofs with a water storage layer," Ecol. Eng., 2016.

[29] A. Palla, I. Gnecco, and L. G. Lanza, “Unsaturated 2D modelling of subsurface water flow in the coarse-grained porous matrix of a green roof," J. Hydrol., 2009.

[30] R. Pitt and J. Voorhees, "The use of WinSLAMM to evaluate the benefits of low impact development.," Proceedings of the Low Impact Develop- ment Conference: Putting the LID on SWM, College Park, MD, USA. pp. 1-2, 2004.

[31] C. F. Chen, M. Y. Sheng, C. L. Chang, S. F. Kang, and J. Y. Lin, "Application of the SUSTAIN model to a watershed-scale case for water quality management," Water (Switzerland), 2014.

[32] A. Abbas et al., "Modelling data of an urban drainage design using a Geographic Information System (GIS)database," J. Hydrol., 2019.

[33] O. US EPA, "Storm Water Management Model (SWMM)," 2018.

[34] D. J. Rosa, J. C. Clausen, and M. E. Dietz, "Calibration and Verification of SWMM for Low Impact Development," J. Am. Water Resour. Assoc., 2015.

[35] Z. Peng and V. Stovin, "Independent Validation of the SWMM Green Roof Module," J. Hydrol. Eng., 2017.

[36] U. S. D. of A. (USDA), "SWMS-2D Model: USDA ARS," 2016. [Online]. Available: https://www.ars. usda.gov/pacific-west-area/riverside-ca/us-salinity-laboratory/docs/swms-2d-model/. [Accessed: 13-Dec-2018].

[37] B. Tomicic and H. S. Andersen, "Storm Water Runoff from Green Urban Areas Modellers' Guideline," Melbourne, Australia, 2015.

[38] M. Just Kjølby, "Modellering af LAR anlaeg samt modellering af stoffjernelse i LAR anlaeg og bassiner Background \&amp; Objective."

[39] P. \&amp; Associates, "WinSLAMM User's Guides." [Online]. Available: http://www.winslamm.com/ select_documentation.html. [Accessed: 09-Dec2018].

[40] O. US EPA, "System for Urban Stormwater Treatment and Analysis Integration (SUSTAIN)," 2016. 